\title{
THE EFFECTS OF TELMISARTAN NEUROPROTECTION ON STROKE WITH HYPERTENSION
}

\author{
Iin Ernawati ${ }^{1}$, Hanik Badriyah Hidayati ${ }^{2}$, Sumarno ${ }^{3}$ \\ Correspondence: iinernawati.apt@gmail.com \\ ${ }^{1}$ Magister of Clinical Pharmacy, Pharmacy Faculty of Airlangga University, Surabaya, Indonesia; \\ ${ }^{1}$ Pharmacy Academy of Surabaya, Surabaya, Indonesia \\ ${ }^{2}$ Department of Neurology, Medical Faculty of Airlangga University, Surabaya, Indonesia. \\ ${ }^{3}$ Department of Clinical Pharmacy, Pharmacy Faculty of Airlangga University, Surabaya, Indonesia.
}

Article History:

Received: March 28, 2018

Accepted: 20 Desember 2019

Published: January 1, 2020

\section{Cite this as:}

Ernawati I, Hidayati $H B$,

Sumoarno. The effects of

telmisartan neuroprotection on

stroke with hypertension. Malang

Neurology Journal; 2020.6:41-

46.

http://dx.doi.org/10.21776/ub.mnj

.2020 .006 .01 .9

\section{ABSTRACT}

Stroke is the second deadly disease in the world after ischemic heart disease. According to data of RISKESDAS (Riset Kesehatan Dasar), stroke was the highest cause of death in Indonesia in 2013. Hypertension is the one of the most important risk factors for stroke. Hypertension therapy is done by modification and the use of antihypertensives. The antihypertensives used is Telmisartan which is a class of Angiotensin Receptor Blocker (ARB) that works by inhibiting bind to angiotensin II type receptors that is angiotensin II type 1 receptors (AT-1R) which directly make angiotensin II bind to AT-2R (angiotensin receptor type 2 receptors). Telmisartan has a neuroprotectant effect that works by inhibiting the appearance of inflammatory cytokines, production of ROS (Reactive Oxygen Species), PGE2 (prostaglandin E2) and NMDA (N-Methyl-D-Aspartate) activity. Telmisartan activates PPARgamma (PPAR- $\gamma$ ), which is very useful in carbohydrate and lipid metabolism which directly protect blood vessels. Telmisartan has the advantage of structure and pharmacokinetics that support the effects of nerve protection. Based on lipophilicity and chemical structure, Telmisartan easily penetrates the brain barrier and high affinity to PPAR- $\gamma$, supporting the effects of Telmisartan neuroprotection. Based on pharmacokinetic aspects, telmisartan has the advantage of having a rapid onset that ranges from 30-60 minutes, with $\mathrm{T} 1 / 2$ (half life) elimination is 24 hours. $\mathrm{T} 1 / 2$ elimination for up to 24 hours from Telmisartan is clinically beneficial to improve medication adherence.

Keywords: Telmisartan, Neuroprotection, stroke, antihypertensives

\section{Introduction}

Stroke is the second deadly disease in the world after ischemic heart disease. ${ }^{1}$ In Western population, stroke is the third cause of morbidity and mortality after cancer and IHD (ischemic heart disease)., ${ }^{2,3}$

Antihypertensives can play a role as a neuroprotection in stroke, some of which include ARBs (Telmisartan, Losartan, Eprosartan, valsartan) and Perindopril. ARBs have potential become neuroprotective and therapeutic use in many brain diseases. ARB neuroprotection activity through anti-inflammatory activity and improvement of the effects of apopotosis triggered by glutamate, beta interleukin, the presence of endotoxy bacteria in nerve cells, astrocytes, microglia and cerebrovascular endothelial cells. ${ }^{4}$ Telmisartan is the antihypertensive class of ARB which has the advantage of being PPAR- $\gamma$ agonist compared to other antihypertensive. ${ }^{5}$ The role neuroprotection of Telmisartan in stroke is that affects of the RAAS (Renin Angiotensin Aldosterone System). The Telmisartan blockade of angiotensin II binding to angiotensin II type 1 receptor (AT$1 \mathrm{R})$, directly triggers the angiotensin II binding activity on angiotensin II type 2 (AT-2R) receptor. This activity can produce the effect of vasodilation and regeneration of nerve cells. Telmisartan can inhibit the formation of inflammatory neurotransmitters and cell damage. Epidemiological studies have shown that incidence ischemic stroke can be decreased by Telmisartan. Telmisartan have neuroprotection effects in stroke patients. This review discusses Telmisartan neuroprotective effects on stroke with hypertension.

\section{Discussion}

\section{Telmisartan Epidemiology Study on Stroke}

Stroke, according to AHA/ASA 2013, is a non-traumatic vascular injury to the CNS (central nervous system) that can lead to both focal and global neurological deficits. The diagnosis of stroke is based on anamnesis, clinical, and radiological symptoms in accordance with anatomy and pathology in neuronal and vascular. The location of the lesion can occur in the brain, spinal or retina. ${ }^{6}$

Epidemiological studies have shown that stroke can be prevented by controlling blood pressure. One option of antihypertensive drugs is Telmisartan. Clinical studies have shown that stroke prevention research (PRoFESS) with Telmisartan monotherapy use and other studies of Telmisartan combination with Ramipril. Ramipril Global Endpoint Trial (ONTARGET) have supported the neurovascular protection effects of Telmisartan on stroke 
incidents. The PRoFESS study compared Telmisartan with placebo in 20332 acute ischemic stroke patients. Therapy with Telmisartan ( $80 \mathrm{mg}$ daily) is given immediately after an ischemic stroke and continuing for 2.5 years did not significantly lower the rate of recurrent stroke, but there was a tendency to reduce stroke recurrence $(p=0.23)$. The PRoFESS study group known that may have led to a significant lack of benefits associated with Telmisartan. Another study comparing ramipril with Telmisartan, and combination therapy showed both can reduce the prevalence of stroke recurrence. Furthermore, the TRANSCEND study comparing placebo with Telmisartan in 5926 patients who were intolerant of antihypertensive ACEI (Angiotensin Converting Enzyme Inhibitor) for 56 months known that the percentage of stroke decreased by $17 \%$ in the Telmisartan group, but not statistically significant $(\mathrm{p}=0.136){ }^{7}$

\section{Prevention of Neuronal Death with Telmisartan}

The incident of stroke causes the blood flow to the brain to decrease which later triggers the process of inflammation and damage to nerve cells or neurons. Inflammatory mediators can be release by the inflammatory response. Inflammatory response can be activation of proteases, microglia, astrocytes and the destruction of the blood-brain barrier. $^{8}$ The presence of hypertension alters brain vascularization and cerebrovascular autoregulation. This triggers a chronic hypoxia and susceptibility to stroke. Chronic hypertension leads to the endothelial vascular damage in the brain causing structural abnormalities in the brain. One study shows that if hypertension is untreated, it can cause lower cognition and smaller brain volume. Telmisartan can normalize blood pressure, restore cerebrovascular remodeling, and improve cerebrovascular autoregulation. $^{4}$

From animal studies, it is found that Telmisartan has neuroprotection and anti-inflammatory activity. In rats stroke-induced or stroke resistant spontaneously hypertensive rats (SHR-SRs) studies, it is known that Telmisartan may affect neuroinflammation factors such as decreasing glial fibrillary acidic protein (GFAP), Nacetylglucosamine oligomers (NAGO), raising the matrix metalloproteinase-9 (MMP-9), collagen IV and inflammation. $^{10}$

Telmisartan and ARB drugs in general, based on their mechanism of action affect the mechanism of RAAS, can provide protection of neurons and control blood circulation by inhibition of angiotensin II binds to angiotensin II type 1 receptors that provide the effect of vasoconstriction. ${ }^{4}$ The new receptor angiotensin II is Mas Receptor (MasR) which binds to angiotensin I and angiotensin II metabolites metabolized by angiotensin converting enzyme 2 (ACE2) which is angiotensin 1-7, may have improvement of neurological deficits. ${ }^{11,12}$ The Telmisartan mechanism as neuroprotective acts through several mechanisms such as direct Telmisartan penetration to the brain. This is influenced by Telmisartan pharmacokinetics factors that have the greater lipophilicity than other drugs in the ARB class, thus affecting the ease of entry into the brain barrier. $^{13,14,15}$ Telmisartan can maintain the blood barrier integrity of the brain, causing neuronal damage resulting from macrophage infiltration; thus, other injury factors in the incidence of stroke can be avoided. ${ }^{4}$ Telmisartan neuroprotection may also pass activation of the signaling pathway (Figure 1). The first signaling pathway activation mechanism is neuroprotection from bacterial endotoxin (gram-negative bacteria). Apoptosis and inflammation caused by endogenous bacteria are the results of membrane activation of membrane toll-like receptors (TLRs) such as co-receptor CD14 and TLR4. ${ }^{16}$ Telmisartan reduces the effects mechanism of pro-inflammatory signal transduction to AT1R stimulation and CD14/TLR4, including NF- $\kappa B$ activation (Figure 1). Induction of $\mathrm{NF}-\kappa \mathrm{B}$ prompts an increase in intracellular inflammation cascade with neurotoxic production of IL- $1 \beta$, interleukin- 6 and alpha tumor necrosis factor, an effect associated with oxidative stress. $^{4}$ Telmisartan directly protects the neuronal from inflammation and the pro-apoptosis from excessive IL- $1 \beta$ stimulation. Neuroprotection acts against cell damage due to IL-1 $\beta$. ARB reduces an increased expression of cyclooxygenase-2 (COX-2) and prostaglandin E2 production and its release is induced by concentrations of neurotoxic of IL-1 $\beta$ (Figure 1). ${ }^{4,17}$ In addition, neuroprotective mechanisms contain kinase-controlled extracellular signals 1 and 2 or p38 mitogen-activated protein kinase phosphorylation and NF- $\kappa \mathrm{B}$ activation (Figure 1). Neuroprotection against IL-1 $\beta$-induced neurotoxicity may occur by the reduction of type 1 interleukin-1 receptors and transcription activity of nicotinamide adenine dinucleotide phosphate oxidase-4, reduction of oxidative stress and hydrogen peroxide induced by cyclooxygenase-2, and the effects associated with decreased JNK. ${ }^{4}, 17$

Telmisartan neuroprotection provides protection from glutamate toxicity, thus reducing the injury of neurons produced by N-methyl-D-aspartate receptors (NMDARs) by glutamate (neurotoxic) (Figure 1). ${ }^{4}$ The neuroprotection mechanism works by decreasing expression of NMDARs, reducing glutamate due to activation of caspase-3 proapoptosis, and giving protection via phosphoinositide-3kinase/protein kinase B/Akt/glycogenase Synthase kinase $3 \beta .^{18}$

Telmisartan triggers PPAR- $\gamma$ activation which is very useful in lipid and carbohydrate metabolism that directly protects the vascular. Other beneficial Telmisartan effects include blood vessel protection and increased sensitivity of insulin and glucose metabolism, so the AT1R blockade and the activation of PPAR- $\gamma$ can increase the blood flow to the brain. Inflammation from neurotoxic presence when the activation of CD14/TLR4 and AT1R dominates the activation stimulation of PPAR- $\gamma .{ }^{4}$ The affinity and activity Telmisartan to the PPAR- $\gamma$ is strongly influenced by the Telmisartan structure of the presence of carboxylic groups (Figure 2) which are responsible for the Telmisartan liphophylicity. ${ }^{2}$

Mitochondrial dysfunction accompanied by excessive ROS formation, increased inflammation and apoptosis are caused by the activation of nicotinamide adenine phosphate dinucleotides, increased oxidative stress and reactive oxygen species (ROS). Inflammation can occur due to the cyclooxygenase- 2 activation (increased the prostaglandins inflammatory production), increased concentrations of nitric oxide and diacylglycerol / protein kinase c / kinase which are regulated by stimulation of extracellular signals 1 and 2 pathways. 
This causes an increase intranuclear trafficking of transcription factors. Transcription factors such as nuclear factor-kappa $\beta$, that increase production of neurotoxic cytokines (interleukin-6, tumor necrosis factor alpha, IL-1 $\beta$ and monocyte chemotactic protein) and pro-inflammatory mediators. The decreasing of survival pathways such as the activation of caspase-3, phosphoinositide-3-kinase/protein kinase $\beta / A k t / g l y c o g e n$ Synthase kinase 3 beta pathway, stimulation of the p38 mitogen-activated protein kinase and c-Jun N-terminal Kinase (JNK), it is known that these are pro-apoptotic mechanisms (Figure 1). ${ }^{4}$ Angiotensin II Type 1 Receptor overstimulation obstructs the neuroprotective PPAR $\gamma$ (peroxisome proliferator-activated receptor gamma) (Figure 2). ${ }^{18}$

ARBs can regulate the signaling pathways. AT1Rs overstimulated enhances the signaling of apoptotic and inflammatory. Mechanisms are interaction with membrane receptors and signaling pathways for injury factors interleukin-1beta (IL-1 $\beta$ ), glutamate, Angiotensin II (Ang II) is heading to increase the injury of cell (Figure 1). ${ }^{4}$

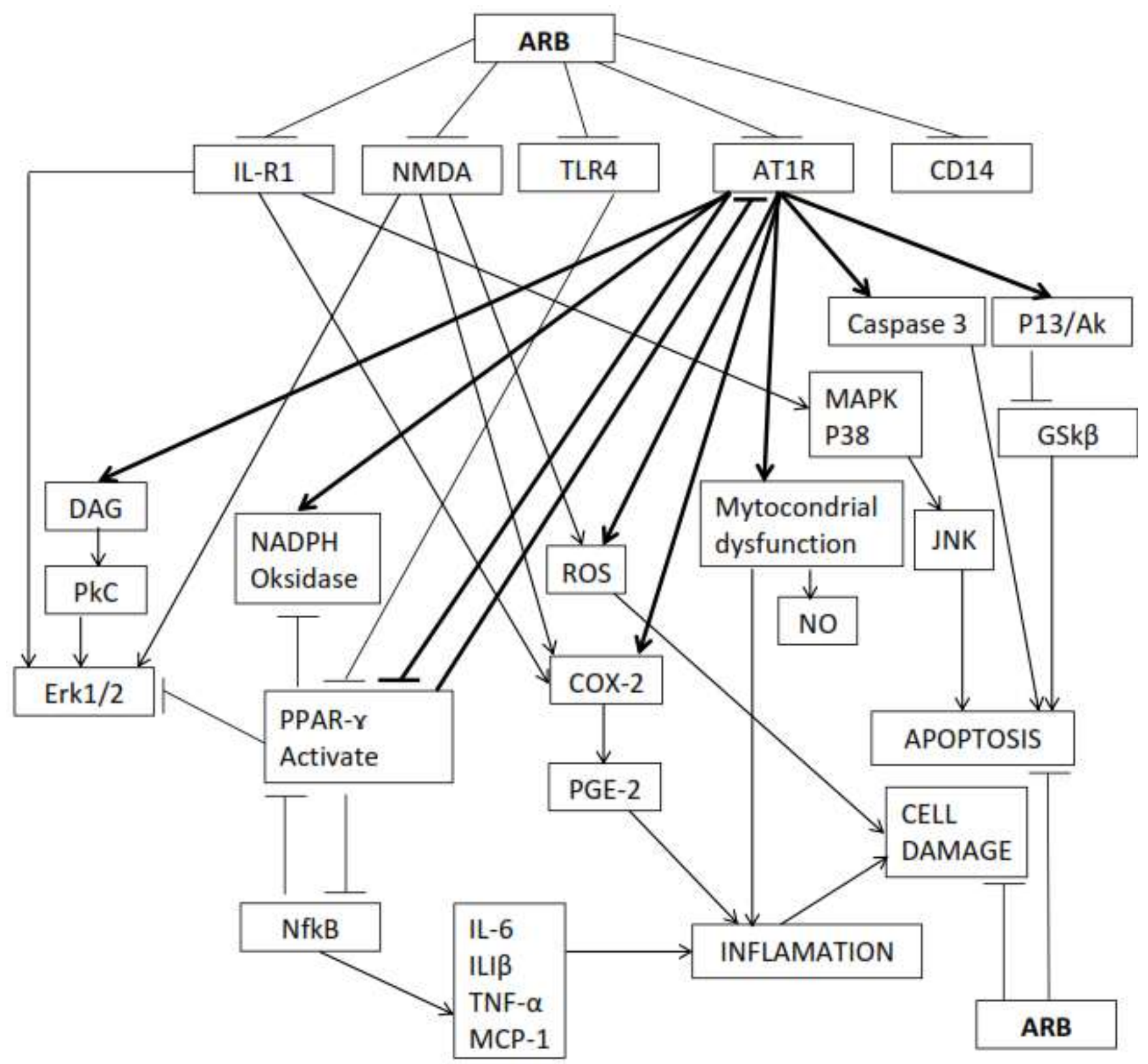

Figure 1. Mechanism of Neuroprotection ARB ${ }^{4}$

Expression of membrane receptors which is cause by activated injury factor (IL-1R1, CD14, TLR4, NMDA) is decreased by ARBs. PPAR $\gamma$ directly stimulated by ARB, even in the absence of AT1R. The stimulation of neuroprotective signaling pathways which follows ARB administration trigger to the decreasing of neuronal injury and inflammation, protection of blood flow and increase energy balance. ARB administration protects cognition, reduces anxiety, stress, depression and may increase lifespan.

Abbreviations: ARB, Angiotensin Receptor Blocker; AT1R, angiotensin II type 1 receptor; IL-1R1, interleukin-1 receptor type 1; NMDA, N-Methyl-D-Aspartate. 


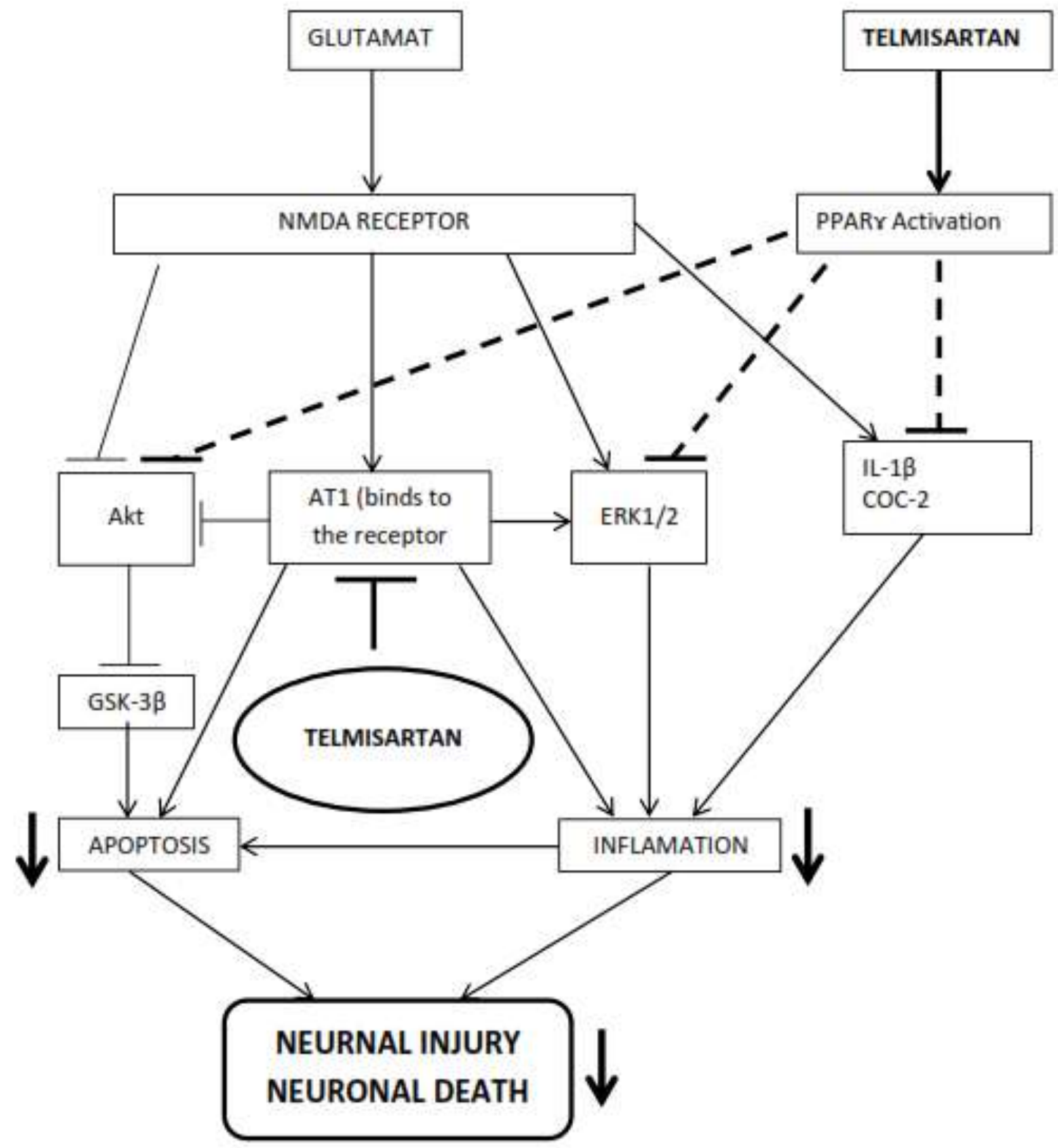

Figure 2. Neuroprotection Mechanism of Telmisartan ${ }^{18}$

NMDA receptors and increase neuronal injury by increasing inflammation and apoptosis activated by Glutamate. Telmisartan decrease glutamate-induced inflammation and apoptosis in cerebellar granule cells (CGCs). Telmisartan neuroprotection is the result of PPAR- $\gamma$ activation and AT1 receptor blockade, decreasing inflamation and apoptosis by mechanisms involving a decreasing of glutamate-induced alterations in the Akt/GSK-3 $\beta$ and ERK1/2 pathways. 


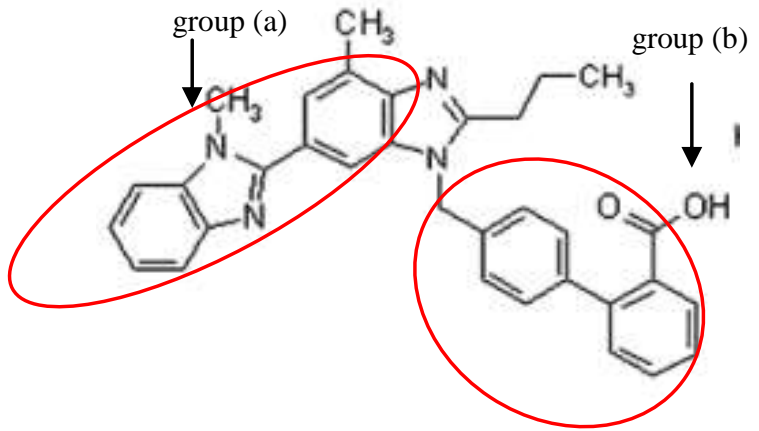

Figure 3. Telmisartan Chemical Structure ${ }^{19}$

(a) is benzyl imidazole group; (b) is carboxyl group

Another mechanism of Telmisartan as neuroprotection is to inhibit HMGB1/RAGE axis. HMGB1 (High-mobility group box 1 protein) play a role in the pathogenesis of various disorders and diseases of organs such as liver, heart, intestine, spine, periodontium, pancreas, lung, and joints, and is involved in transplantation graft rejection. HMGB1 and RAGE (Receptor for Advanced Glycation End Products) contribute to the pathogenesis of hypertension and atherosclerosis. Telmisartan lowers serum sRAGE levels in patients with hypertension. Telmisartan inhibits cellular injury due to AGE-elicited through suppression of RAGE expression. Suppression of RAGE expression occurs by activation of PPAR $-\gamma .^{5}$ Telmisartan inhibition of HMGB1/RAGE axis on stroke risk factors such as atherosclerosis and hypertension may be a neuronal protective pathway against stroke events.

\section{Pharmacokinetic and Chemical Structures Aspects that Affect Activity Neuroprotection of Telmisartan}

Pharmacokinetics factors from the drug such as lipophilicity, half-life, and bioavailability of the drug may affect the activity, duration of the action, and affinity of drug-binding with the receptor. This pharmacokinetics profile is important to guide in choosing the right of ARB and according to the patient's condition. Based on the pharmacokinetics factors, Telmisartan has the highest lipophilicity compared to other ARB classes, this is due to the replacement of tetrazole groups into carboxylate groups, which increases Telmisartan hydrophobicity (Figure 3). ${ }^{19}$ This advantage also allows Telmisartan to penetrate the blood brain barrier. The Telmisartan affinity of PPAR- $\gamma$ is strongly influenced by the Telmisartan structure. Telmisartan stucture presence of carboxylate groups that enhance telmisartan lipophilicity (Figure 3). ${ }^{19}$ It is known that Telmisartan is not a prodrug, so this drug works directly without being metabolized by the first metabolism in liver. ${ }^{20}$

Telmisartan has the fastest onset that ranges from 30 minutes to 1 hour, with $\mathrm{T} 1 / 2$ (half life) elimination reaches 24 hours. This is particularly important in clinical settings, since once-daily use greatly benefits patients to have high adherence. $^{20}$ Telmisartan has the possibility of drug interactions with digoxin, which Telmisartan can increase blood digoxin levels, through P-glycoprotein inhibition mechanisms, dose adjustment in patients with Telmisartan use as antihypertensives and digoxin. ${ }^{13}$ In the metabolic phase, Telmisartan is one of the ARBs not undergoing phase 1 metabolism and not through metabolism with cytochrome $\mathrm{P} 450$, so the possibility of drug interactions with other drugs is very minimal. Penetration of drug and metabolites to the body tissue is also influenced by the presence of substrates that help transporting drugs such as substrate Pgp. ${ }^{15}$

\section{Conclusion}

Neuroprotection of Telmisartan on stroke patients with hypertension by inhibiting the appearance of inflammatory cytokines, ROS production, prostaglandin E2 and NMDA activity and PPAR- $\gamma$ activation.

\section{Funding}

This review article did not receive any financial support.

\section{Conflict of Interest}

The author states there is no conflict of interest.

\section{Acknowledgement}

We thank Prof. Suharjono, MS., Apt. as head of the Airlangga University Clinical Pharmacy Masters program has provided support so that the review of this article can be resolved properly

\section{References}

1. WHO, the top 10 causes of death fact sheet: updated January 2017. Avalaible from: http://www.who.int/mediacentre/factsheets/fs310/en/

2. Saenger, AK, Christenson, RH. Stroke biomarkers: progress and challenges for diagnosis, prognosis, differentiation, and treatment. Clin Chem; 2010.56(1):21-33 DOI: 10.1373/clinchem.2009.133801

3. Raisa, N, Sujuti, H, Purnomo, H, Rahayu, M, Dalhar M. The influence of sampling time to serum glial fibrillary acidic protein (GFAP) levels in acute stroke. Malang Neurology Journal; 2019.5:30-33. DOI: 10.21776/ub.mnj.2019.005.01.

4. Villapol, S and Saavedra, JM. Neuroprotective Effects of Angiotensin Receptor Blockers, American Journal of Hypertension; 2015.28(3). DOI: 10.1093/ajh/hpu197

5. Kikuchi, K, Tancharoen, S, Ito, S. Review Potential of the Angiotensin Receptor Blockers (ARBs) Telmisartan, Irbesartan, and Candesartan for Inhibiting the HMGB1/RAGE Axis in Prevention and Acute Treatment of Stroke, Int. J. Mol. Sci; 2013. 13: 18899-18924. DOI: 10.3390/ijms140918899

6. Cheung RTF. A Systematic Approach to the Definition of Stroke. Austin J Cerebrovasc Dis \& Stroke; 2014.1:5;1024. Avalaible from: https://austinpublishinggroup.com/cerebrovasculardisease-stroke/fulltext/ajcds-v1-id1024.pdf

7. Sik Hong, K. Blood Pressure Management for Stroke Prevention and in Acute Stroke. Journal of Stroke; 2017.19(2):152-165. DOI: 10.5853/jos.2017.00164

8. Setianto, CA, Purnomo, H, Marhaendraputro, EA, Santoso, WM, Correlation Of C-Reactive Protein 
Levels With Clinical Outcome Intracerebral Hemorrhage Stroke Patients. Malang Neurology Journal; 2015;1(1):23-27. DOI: 10.21776/ub.mnj.2015.001.01.5

9. Chrismanita, A, Ong, Rizal, A. Hubungan antara Kejadian Hipertensi dengan Performa Fungsi Kognisi dan Gambaran Magnetic Resonance Imaging Kepala, Neurona; 2015. Vol 32 no. 2.

10. Kono, S, Kurata, T, Sato, K, Omote Y, Hishikawa N, Yamashita T, Deguchi K, Abe K. Neurovascular Protection by Telmisartan via Reducing Neuroinflammation in Stroke Resistant Spontaneously Hypertensive Rat Brain after Ischemic Stroke. "Journal of Stroke and Cerebrovascular Diseases", Article in Press; 2014. 1-11. DOI: 10.1016/j.jstrokecerebrovasdis.2014.09.037

11. Sumners, C, Horiuchi, M, Widdop, R, McCarthy C, Unger T, Steckelings UM. Evolving Concepts of the Renin-Angiotensin System Protective arms of the renin-angiotensin-system in neurological disease, Clinical and Experimental Pharmacology and Physiology; 2013.40: 580-588. DOI: 10.1111/14401681.12137

12. Arroja, M, Reid, E, Mc Cabe, M. Review: Therapeutic potential of the rennin angiotensin system in ischaemic stroke, Experimental \& Translational Stroke Medicine; 2016.8:8. DOI: 10.1186/s13231016-0022-1

13. Burkey, K, Hoffmann, JC, Nemerovski, S. et al., Drug Information Handbook. A Clinically Relevant Resource for all healthcare Professionals. American Pharmacist Association, 24 ${ }^{\text {th }}$ Edition, Ohio: LexiComp Inc; 2015.

14. Al Sabbah, Z, Mansoor, A, Kaul, U. Angiotensin Receptor Blockers - Advantages of the New Sartans, Journal of The Association of Physicians of India; 2013. Jul;61(7):464-70. Avalaible from: https://www.ncbi.nlm.nih.gov/pubmed/24772750
15. Michel, MC, Foster, C, Brunner, HR, Liu, L. A systematic comparison on the properties of clinically used angiotensin II type 1 receptor antagonists. Pharmacol Rev, 2013; 65:809-848. DOI: 10.1124/pr.112.007278

16. Benicky, J, Sánchez-Lemus, E, Honda, M, Pang T, Orecna M, Wang J, Leng Y, Chuang DM, Saavedra JM. Angiotensin II AT1 receptor blockade ameliorates brain inflammation. Neuropsychopharmacology; 2011.36:857-870. DOI: $10.1038 / \mathrm{npp} .2010 .225$

17. Pang, T, Wang, J, Benicky J, Sanchez-Lemus E, Saavedra JM. Telmisartan directly ameliorates the neuronal inflammatory response to IL-1 $\beta$ partly through the JNK/c-Jun and NADPH oxidase pathways. J Neuroinflammation; 2012.9:102 DOI: $10.1186 / 1742-2094-9-102$

18. Wang, J, Pang, T, Hafko, R, Benicky J, SanchezLemus E, Saavedra JM, Telmisartan ameliorates glutamate-induced neurotoxicity: roles of AT(1) receptor blockade and PPAR- $\gamma$ activation", Neuropharmacology; 2014.79:249-261. DOI: 10.1016/j.neuropharm.2013.11.022

19. Kakuta, H, Kurosaki, E, Niimi, T. Gato K, Kawasaki Y, Suwa A, Honbou K, Yamaguchi T, Okumura H, A Distinct Properties of Telmisartan on Agonistic Activities for Peroxisome Proliferator-Activated Receptor $\gamma$ among Clinically Used Angiotensin II Receptor Blockers: Drug-Target Interaction Analyses". J Pharmacol Exp Ther, 2014; 349:10-20. DOI: $10.1124 /$ jpet.113.211722

20. Abraham, HM, White, M, White, W. The Comparative Efficacy and Safety of the Angiotensin Receptor Blockers in the Management of Hypertension and Other Cardiovascular Diseases. Drug Saf; 2015. 38:1: 33. DOI: 10.1007/s40264-014023 